\title{
Research on Positioning Detection Device of Coordinate Measuring Machine
}

\author{
Kui Jin \\ Dalian Vocational \& Technical College Dalian, China \\ jk_810912@163.com
}

Keywords: positioning device design; positioning accuracy; install efficiency

\begin{abstract}
It is a technical problem which need to be solved urgently that how the coordinate measuring machine tool ensure the accuracy of the relocating accuracy and the efficiency of clamping detection when batch testing the parts of bearing housing. According to the characteristics of the parts of bearing housing, this paper design an adjustable positioning device to solve problems such as: positioning problems of workpiece, low detection accuracy and the problem of low efficiency of work when batch testing the parts of bearing housing. Finally, the designed device is applied to the actual test and the above problems encountered in the production are solved.
\end{abstract}

\section{Introduction}

As the rapid development of mechanical processing technology, mechanical automation has the trend of replacing traditional manual production. The Coordinate measuring machine(CMM) is an indispensable machine tools in modern precision machinery inspection and processing. As the rapid development of modern technology, the applying of CMM in machine work is becoming more and more worldwide [1]. The three-dimensional contour and shape measurement of objects have been widely used in mechanical manufacturing, aerospace, navigation, reverse engineering, transportation, power, medical, military, telecommunications and other fields [2]. With the continuous research of measurement methods, it is also an increasing demand for three-dimensional coordinates measurement technology. The related enterprises have also conducted in-depth study on how to improve the measurement efficiency and reduce the labor intensity in the process of using three-coordinate system [3].

In this paper, the auxiliary tools are used to test the parts of bearing pedestal in batch and the reasonable utilization of auxiliary tools can not only improve the detection accuracy and efficiency of production, but also greatly reduce the labor intensity of the employees.

\section{Traditional Concentricity Testing Method of Bearing Pedestal}

Tests of concentricity tolerance and geometric tolerance are very common in product components measurement. Special inspection tools and composite accessories such as: V type bracket, offset pendulum and Steel ball and leverage percentage chart are used to test regular axis parts. The concentricity tolerance of box hole type Parts are often tested by Core-axis plus lever meter or roundness tester. It is very difficult to test the concentricity tolerance of irregular bore in some large parts in regular way [4]. In the early stage of bearing pedestal inspection, it is common to use the auxiliary testing instruments to measure the concentricity of both ends of bearing pedestal and the perpendicularity between the both ends of bearing pedestal and the axis of the bearing pedestal. The test results will be easily affected by its complex testing procedures, auxiliary testing instruments and the testing personnel. The profile projector is used when problems are found in the early stage of bearing pedestal inspection [5]. Due to the different understanding and different testing methods of reference axis, the different evaluation methods of concentricity tolerance and the mining error of profile projector, when profile projector is used to measure the concentricity, the error of measurement is large and the repeatability is poor, which means the measurement results cannot reflect the true concentricity tolerance of the parts. Therefore the coordinate measuring machine (CMM) is a good choice in testing concentricity tolerance and geometric tolerance. Compared with 
professional concentricity tolerance measuring instruments, there are many advantages of CMM such as: the workpiece do not need to be rotated, special core-axis or V-type bracket are not needed, no need for machinery alignment. The measurement results can be obtained quickly by sampling the workpiece with the probe. Using the coordinate measuring machine can guarantee the accuracy of the coaxial degree of the inner hole of the bearing pedestal, but the detection efficiency is relatively low for the batch detection and the accuracy loss of the machine tool is large.

According to the technical problems above, the purpose of this paper is to design a new and simple positioning device for CMM to solve the problems such as inaccuracy in mass detection of bearing pedestal, the detection accuracy is not high and the work efficiency is low. The mechanism of this device is simple, compared with the prior detection method, the process, safety and economy is better and the cost is lower and the clamping is simple and flexible. It solves the problem of mass measuring the concentricity and the perpendicularity in 0.01of the inner hole of the bearing pedestal in both ends, which is shown as Fig. 1 and Fig.2.
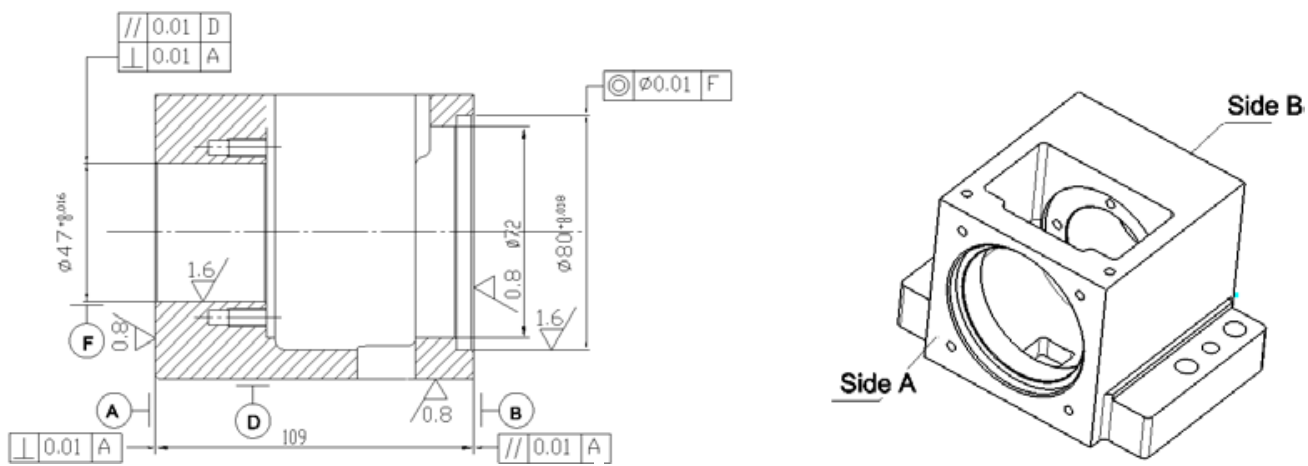

Fig.1 Diagram of type I bearing pedestal parts
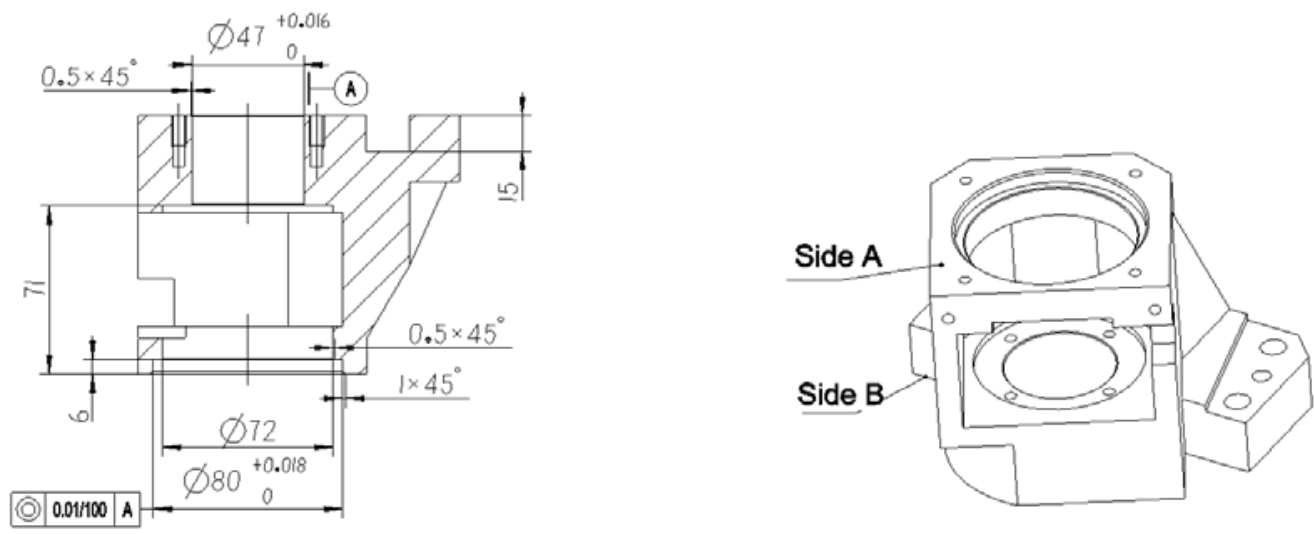

Fig.2 Diagram of type II bearing pedestal parts

\section{The Structure of the CMM Positioning Detection Device}

The CMM positioning detection device is composed of the following compartments: 1 T-shaped block, 2bolt, 3 vertical plate ,4 counterweight block, 5 bolt, 6 cushion block, 7 bottom board, 8 bolt, 9 pin, 10 gasket, 11 bolt, 12 bolt, 13 pin, 14 chuck, 15 type I bearing housing, 16 bolt, 17 pin, 18 gasket, 19 type II bearing housing.

The feature is: put two cushion blocks (6) on the bottom board (7), positioned and fastened with blot (8). Then the fixed bottom board (7) with two cushion blocks (6) , vertical plate (3) will be fixed on chuck (14) by blot (2) and T-shaped block (1). The bottom face of type I bearing pedestal (15) is placed on cushion block (6) and positioned with pin (9) then fastened by blot (11) with gasket (10). The upper surface of cushion block (6) is in contact with the datum plane of type I bearing pedestal (15). In order to remove the stains of the cushion block and maintain a good 
contact surface, the surface of cushion block (6) has a groove and 1mm higher than the base plate for accurate positioning (As shown in Fig.3). When type II bearing pedestal is inspected, remove cushion block (6) and bottom board (7).Type II bearing pedestal is in contact with vertical plate, then positioned by pin (17) and fastened by blot (16) with gasket (18) (As shown in Fig. 4) .

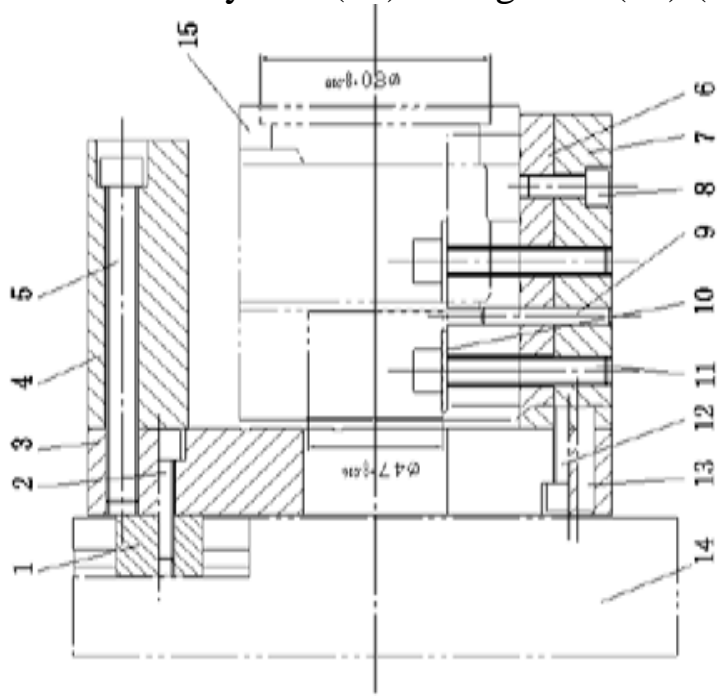

Fig.3 Type I bearing pedestal clamping diagram 1-T-shaped block, 2-bolt, 3-vertical plate, 4-counterweight block, 5-bolt, 6-cushion block, 7-bottom board, 8-bolt, 9-pin, 10-gasket, 11-bolt, 12-bolt, 13-pin, 14-chuck, 15-type I bearing housing

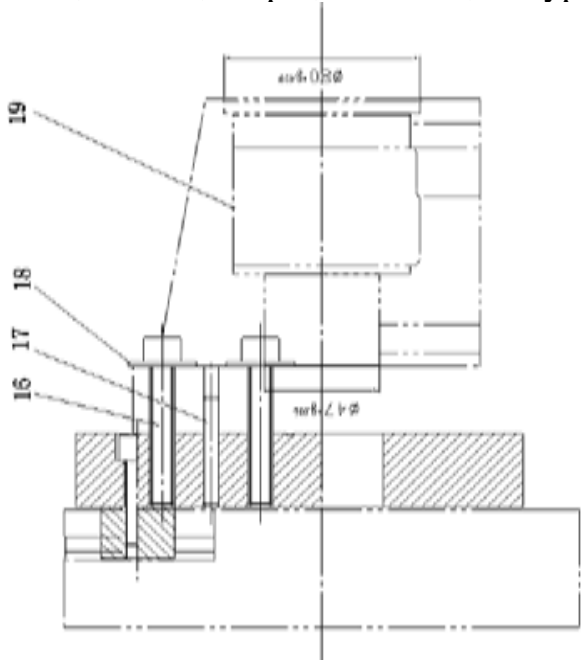

Fig.4 Type II bearing pedestal clamping diagram 16- bolt, 17- pin, 18-gasket, 19-type II bearing housing

\section{The Instruction of the CMM Positioning Detection Device}

When type I bearing housing is tested, the upper surface of cushion block is in contact with the datum plane of type I bearing housing. In order to remove the stains of the cushion block and maintain a good contact surface, the surface of cushion block has a groove(depth $0.5 \sim 2 \mathrm{~mm}$ ) and $1 \mathrm{~mm}$ higher than the base plate. Two cushion blocks will be fixed on bottom board and fastened with blot. Then this bottom board with fixed cushion blocks and vertical plate will be fixed on chuck by blot and T-shaped block. Tighten the bolt after adjusting the center position. The bottom face of type I bearing housing is placed on cushion block and positioned with pin then fastened by blot with gasket.

The detection process is to install the bearing housing on the fixed positioning device then measure the concentricity of the two bores and the geometric tolerance between axis and end face of the two bores.

When type II bearing housing is inspected, remove cushion block and bottom board. Type II bearing housing is in contact with vertical plate, which has a groove (depth $0.5 \sim 2 \mathrm{~mm}$ ) in order to 
remove the stains of the vertical plate and maintain a good contact surface. Then type II bearing housing is positioned by pin and fastened by blot with gasket (As shown in Fig.5) .

The detection process is to install the bearing housing on the fixed positioning device then measure the concentricity of the two bores and the geometric tolerance between axis and end face of the two bores.

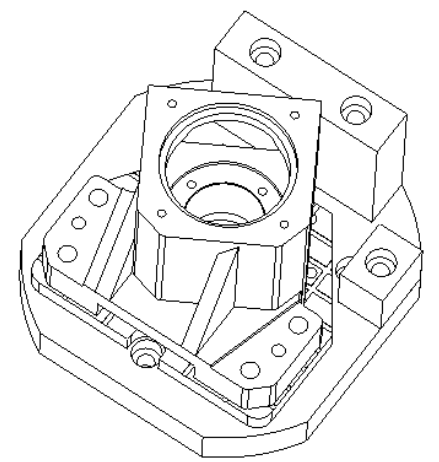

Fig. 5 Detection and positioning device diagram

\section{Conclusion}

The designed CMM positioning device has been proved by repeated inspection and use in the field and solved the clamping problem in mass quality inspection of the bearing housing. This device can reduce the production cost, save the detection time, greatly improve the work efficiency and get the user's approval. It is proved by practice that the detection positioning device is simple structured. Compared with the traditional detection method, it is better in economy, process and production cost. The clamping is flexible and simple, which solves the problem that the workpiece repeatedly locates without overlap and the low detection efficiency in mass testing the bearing pedestal parts.

\section{References}

[1] Xu hao. Mechanical Design Manual [K]. Machinery Industry Press,1995

[2] Liu Shouyong, Machine Manufacturing Process and Machine Tool Fixture [M]. Mechanical Industry Press, 2008.

[3] Li Changnian Design and manufacture of fixture for machine tool [M]. Beijing: Machinery Industry Press, 2007

[4] Lin Wenhuan Fixture Design for machine tools [M]. Beijing: National Defense Industry Press, 2004.

[5] Feng Bingrao, Wang Nangen, Wang Xiaoxiao Brief handbook of mould design and manufacturing [M]. Shanghai: Shanghai Science and Technology Press, 2015. 